\title{
An observational study of fixed-dose Tanacetum parthenium nutraceutical preparation for prophylaxis of pediatric headache
}

Filomena Moscano ${ }^{1}$, Michela Guiducci ${ }^{2}$, Lucia Maltoni ${ }^{1}$, Pasquale Striano ${ }^{3}$, Maria Giuseppina Ledda ${ }^{4}$, Francesco Zoroddu ${ }^{5}$, Umberto Raucci $^{6}$, Maria Pia Villa ${ }^{2}$ and Pasquale Parisi ${ }^{2^{*}}$ (])

\begin{abstract}
Background: Migraine is one of the most prevalent chronic pain manifestations of childhood. Despite the multitude of available treatments, parents are often concerned about chronic therapies and pediatricians have insufficient confidence in prescribing prophylactic drugs. Therefore, there is now growing interest for natural supplements used to control recurrent migraine headaches. Such approach may increase acceptance and adherence to long-term prophylaxis therapy in children.
\end{abstract}

Methods: This is an observational multicenter study performed in children $(n=91)$ with migraine, with $(\mathrm{MO})$ or without aura (MA), or tension-type headache (TTH). A fixed-dose Andrographis paniculata, CoQ10, riboflavin, and magnesium, was administered for 16 weeks. Patients were evaluated at baseline (T0), at week 8 (T1) and at the end of treatment at week 16 (T2). A follow-up period occurred at week 20 (T3) and week 32 (T4).

Results: The herbal supplement significantly reduced the frequency of headaches in $\mathrm{TTH}$ patients during treatment period (TO: $11.97 \pm 1.92$ vs T2: $5.13 \pm 1.93 ; p<0.001$ ) and the efficacy was maintained after 16 weeks of treatment withdrawal (T4: 4 . $46 \pm 1.75 ; \bar{p}<0.001$ vs T0). The frequency of migraine attacks was also reduced in the MO group during treatment (TO: 9. $70 \pm 0.96$ vs T2: $4.03 \pm 0.75 ; p<0.01)$ and after withdrawal (T4: $2.96 \pm 0.65 ; p<0.01$ vs T0). Conversely, MA patients showed reduction in migraine's frequency during treatment (T0: $8.74 \pm 1.91$ vs T2: $3.78 \pm 2.02 ; \mathrm{p}<0.01$ ) but not at the end of the study (T4: $5.57 \pm 3.31 ; p>0.05$ vs T0).

$\mathrm{TTH}$ patients did not report significant improvement of pain intensity. A significant effect was observed in the MO group during treatment (T0: $3.06 \pm 0.11$ vs T2: $2.14 \pm 0.19 ; p<0.001)$ and after treatment withdrawal $(T 4: 2.20 \pm 0.21 ; p<0.001$ vs T0). Likewise, MA group shōwed a significant treatment effect (T0: $2.57 \pm 0.20$ vs T2: $0.86 \pm 0.45 ; p<0.001$ ) and the efficacy persisted at the end of the study (T4: $1.00 \pm 0.58 ; \mathrm{p}<0.001 \mathrm{vs}$ T0).

Conclusion: This fixed-dose Tanacetum parthenium preparation improved headache frequency and pain intensity in children affected by TTH. Despite the main limits, this study supports the use of nutraceutical in pediatric headache/ migraine.

Keywords: Nutraceuticals, Pediatric migraine, Observational study, Tanacetum parthenium, Prophylaxis

\footnotetext{
* Correspondence: pasquale.parisi@uniroma1.it; parpas@iol.it

${ }^{2}$ Child Neurology, Pediatric Headache \& Sleep Disorders Centre, Chair of

Pediatrics, NESMOS Department, Faculty of Medicine and Psychology,

Sapienza University, Via Di Grottarossa, 1035-1039, 00189 Rome, Italy

Full list of author information is available at the end of the article
}

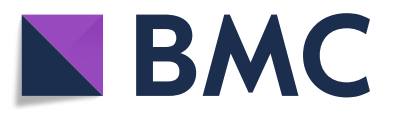

(c) The Author(s). 2019 Open Access This article is distributed under the terms of the Creative Commons Attribution 4.0 International License (http://creativecommons.org/licenses/by/4.0/), which permits unrestricted use, distribution, and reproduction in any medium, provided you give appropriate credit to the original author(s) and the source, provide a link to the Creative Commons license, and indicate if changes were made. The Creative Commons Public Domain Dedication waiver (http://creativecommons.org/publicdomain/zero/1.0/) applies to the data made available in this article, unless otherwise stated. 


\section{Background}

Migraine is one of the most prevalent neurological symptom and chronic pain manifestation of childhood, affecting up to $10 \%$ of children between the ages of 5 and 15 years and up to $28 \%$ of adolescents aged from 15 to 19 years [1]. Migraine is defined as an episodic pain disorder that can vary from an occasional occurrence to a daily frequency and can subsequently become a very disabling disorder, with a substantial effect on the child's quality of life $[2,3]$. The disease can cause a significant distress as for the child, as for the entire family. If not successfully treated, frequent and recurrent headaches can impact families with significant disability due to loss of school, work, and social activities. As for adults, migraine treatment traditionally includes acute therapy for aborting migraine attacks and prophylactic treatment for reducing the frequency, duration and severity of attacks [4]. Notably, headache amongst children has a high risk of development into a chronic condition and persisting into adulthood [5]. Thus, in children an effective early treatment would be expected to impact disease progression before many of the refractory aspects seen in adults become established [6]. There are several classes of medications that may be used for prophylactic therapy like antidepressants, anticonvulsants, antihistamines, beta-adrenergic receptor blockers, and calcium ion channel antagonists, botulinum toxin $[7,8]$. Due to the potential limitations of conventional treatment of obtaining a satisfactory response to pain in many cases of primary headache, various alternative treatments are sought by patients; in particular, parents ask healthcare professionals to provide migraine relief for their children. Moreover, even clinicians prefer to avoid prescription of prophylactic therapies in children due to the poor evidence of efficacy and significant potential adverse effects in this population [9] and the well-known "placebo effect" observed in children [10].

There is now growing interest for natural supplements for migraine and headache treatment. Due to the low side effects, several studies support the efficacy of natural treatments, in particular nutraceuticals, in the prophylactic treatment of migraine in children and adolescents, even if the evidences are still limited [11].

Nutraceutical is a term derived from "nutrition" and "pharmaceutical", indicating "food, or parts of a food, that provide medical or health benefits, including the prevention and treatment of diseases". Nutraceutical treatment consists of taking vitamins, supplements (like magnesium, riboflavin, coenzyme Q10, and alpha lipoic acid) and herbal preparations (like feverfew and butterbur). This type of approach enhances patient acceptance and adherence to long-term prophylaxis therapy combined with appropriate information on treatments' efficacy and safety [11].

Magnesium $\left(\mathrm{Mg}^{2+}\right)$ has a role in various biological processess and it is involved in ATP production and function and in the control of vascular tone and it binds to NMDA receptors; its role has been suggested because of magnesium deficiency demonstrated in headache physiopathogenesis [11]. Coenzyme Q10 (CQ10) has shown a crucial role in sustaining mitochondrial energy stores, as a carrier in the mitochondrial electrons transport chain; furthermore it has antioxidant proprieties. A role of Cq10 in migraine pathogenesis has been postulated because migraineurs have difficulties in energy production [11]. Riboflavin (B2 vit) has two active coenzyme forms cofactors in oxidation-reduction reaction of flavoproteins and seems to reduce symptoms in patients with mitochondrial dysfunction [11]. The anti-migraine activity of Tenacetum Partheniunim (Feverfew) is probably related to the inhibition of oxide nitric synthesis, to the cytokines induction, to the release of serotonin from the platelets and to the inhibition of the Calcitonin Gene-Related Peptide (CGRP) release from the trigeminovascular system [11].

To this aim, keeping in mind all the above reported considerations, we have performed a multicenter, prospective study to evaluate the efficacy and safety of Partena ${ }^{\circledR}$ tablets (a combination of $\mathrm{Mg}^{2+} 169 \mathrm{mg}$, CoQ10 20 mg, VitB2 4,8 mg, Feverfew 150 mg-1,2 mg Parthenolides and Andrographis paniculata $100 \mathrm{mg}$ ) available in Italy as a dietary supplement (Italian Registry of Supplements code 64289) in a population suffering from migraine/ headache in the developmental age, referring to headache outpatient centers.

\section{Methods}

\section{Study design}

This was a multicenter, prospective, observational study in children and adolescents with migraine (with or without aura) or tension-type headache. From January 2016 to December 2017, patients from 9 to 18 years were consecutively enrolled from five different neurologic pediatric centers in Italy (Sapienza University-Sant'Andrea Hospital, Rome, Gaslini Childrens' Hospital, Genoa, Sant'Orsola-Malpighi Hospital, Bologna, "Businco Hospital", Cagliari, and University Hospital, Sassari). The study was conducted in a context of routine practice without any additional or unusual procedure of diagnosis or surveillance. The study was also conducted according to the Declaration of Helsinki. Written informed consent from a parent or guardian and, when appropriate, child assent were obtained prior to study partecipation. Patients underwent a baseline evaluation performed by Pediatricians or Child Neurologists with expertise in pediatric headache, in order to confirm the diagnosis and their eligibility for the study. During the assessment patients reported their age, medical history, migraine family history, headache type and localization. Inclusion criteria were migraine with aura (MA), without aura (MO) or tension-type headache (TTH), as defined according to the International Classification of Headache Disorders, 3rd 
Edition [12]. Moreover, a headache frequency of 4 or more days/month should have been reported on a headache diary over a pre-baseline period, for at least the latest 3 months. Patients were excluded if they previously used headache prophylactic treatment (drugs, nutritional supplement or psychotherapy); all secondary headache disorders were also excluded.

Partena ${ }^{\oplus}$ tablets, 1 tablet, twice a day, at regular interval, was administered by Parents' children, for the first 4 weeks, following by 12 -weeks constant-dose phase of 1 tablet per day. Adherence and compliance to therapy and side effects were recorded in the clinical headache diary, during all phases of study. Patients were evaluated at baseline (T0) at week 8 (T1) and at the end of treatment at week 16 (T2). At week 20 (T3) and week 32 (T4), each enrolled patient was re-evaluated (follow-up period). Obviously, before entering the baseline phase, children had to meet inclusion and exclusion criteria, as specified above.

\section{Pain assessment}

Patients completed a daily headache diary, in accordance with the NINDS Common Data Elements [13]. A headache day was defined as any day during which a headache occurred within a 24-h period, starting at midnight. The four-point pain scale (none, mild, moderate, severe) has been used in this study to assess treatment efficacy.

\section{Safety}

Safety was assessed with the use of adverse-event reports that were collected from parents and patients by means of a structured interview.

\section{Statistical analysis}

A paired analysis of variance (ANOVA) and post-hoc paired t-test analysis was performed to analyze comparison during treatment and follow-up period. Data were given as mean \pm standard deviation (SD). The two-sided Fisher exact test was performed for contingency analysis. Patients who did not maintain compliance to treatment of at least $85 \%$ till the end of the study were considered as censored data for the "pre-protocol" analysis. We accepted as significant $p$ values of less than 0.05 .

\section{Results}

A total of 91 patients (mean age 14.4 \pm 2.2 ; males $34 \%$ and females 66\%) were enrolled (demographic and clinical variables at baseline are shown in Table 1.). Treatment with Partena was substantially well tolerated, but $4.4 \%$ of patients interrupted the treatment due to gastrointestinal symptoms (nausea and diarrhea). A small group of patients $(8.8 \%)$ interrupted the study protocol due to treatment inefficacy while the $7.7 \%$ of patients was lost at follow-up.
Table 1 Demographic and clinical characteristics of patients at baseline $(n=91)$

\begin{tabular}{ll}
\hline Variable & mean $( \pm$ s.d.) or $\mathrm{n}(\%)$ \\
\hline Age, years & $14,4 \pm 2,2$ \\
Female & $60(66 \%)$ \\
Male & $31(34 \%)$ \\
Tension-type Headache (TTH) & $20(22 \%)$ \\
Migraine with aura (MA) & $7(8 \%)$ \\
Migraine without aura $(\mathrm{MO})$ & $64(70 \%)$ \\
\hline
\end{tabular}

In the intention-to-treat analysis $(n=91)$ the percentage of patients showing a significant reduction of $50 \%$ or more in the number of headache episodes at the end of the 16-weeks treatment period (T2) was $50 \%$ in the TTH group, $55 \%$ in the $\mathrm{MO}$ group and $85 \%$ in the MA group. A significant maintenance of this effect after 16 weeks from the treatment withdrawal (T4), was seen for TTH (50\%) and MO group (53\%), but not for MA patients which showed a slight but significant reduction in the efficacy respect to the treatment period $(71 \%, p<0.01$ respect to T2).

The percentage of patients who completed the study was $75 \%$ of TTH, $83 \%$ of MO group and $100 \%$ of MA.

In the analysis per-protocol $(n=72)$, Partena significantly reduced the frequency of headaches respect to baseline in TTH patients $(\mathrm{F}(1.574,22.04) ; p=0.004)$ during the period of treatment assumption (T0: $11.97 \pm 1.92$ vs T2: $5.13 \pm 1.93 ; p<0.001)$. The efficacy was maintained even after 16 weeks of treatment withdrawal (T4: $4.46 \pm 1.75$; $\mathrm{p}<$ 0.001 respect to T0). A significant reduction of migraine frequency was also found for MO group $(\mathrm{F}(2.740,131.5) ; p<$ 0.0001 ) both during treatment (T0: $9.70 \pm 0.96$ vs T2: $4.03 \pm 0.75 ; p<0.01)$ and after 16 weeks of treatment withdrawal (T4: $2.96 \pm 0.65 ; p<0.01$ respect to T0). Conversely, MA patients showed a significant effect in reducing headache frequency during treatment (T0: $8.74 \pm 1.91$ vs T2: $3.78 \pm 2.02 ; p<0.01$ ) but not until the end of the study (T4: $5.57 \pm 3.31 ; p>0.05$ respect to T0). (Fig. 1a).

In the assessment of pain intensity, TTH patients did not show any significant amelioration (F (2.463, 34.49); $p>0.05)$. Interestingly, a significant effect of treatment was found in $\mathrm{MO}$ group $(\mathrm{F}(3.471,166.6) ; \mathrm{p}<0.0001)$. In particular, a reduction of pain intensity was found since 8 weeks of treatment (T0: $3.06 \pm 0.11$ vs T1: $2.31 \pm 0.17 ; p$ $<0.001$ ), remain significant at 16 weeks (T2: $2.14 \pm 0.19 ; p$ $<0.001$ respect to T0) and at follow-up visits after treatment withdrawal (T4: $2.20 \pm 0.21 ; p<0.001$ respect to T0). Similarly MA group showed a significant treatment effect $(\mathrm{F}(1.459,8.752) ; \mathrm{p}<0.01)$ with a good response since 8 weeks of treatment (T0: $2.57 \pm 0.20$ vs T1: $1.00 \pm 0.38 ; \mathrm{p}$ $<0.001$ ), after 16 weeks (T2: $0 . \overline{86} \pm 0.45 ; p<0.001$ respect to T0) and the efficacy was maintained until the end of the study (T4: $1.00+0.58 ; p<0.001$ respect to T0) (Fig. 1b). Globally, the reduction of pain intensity was higher, 

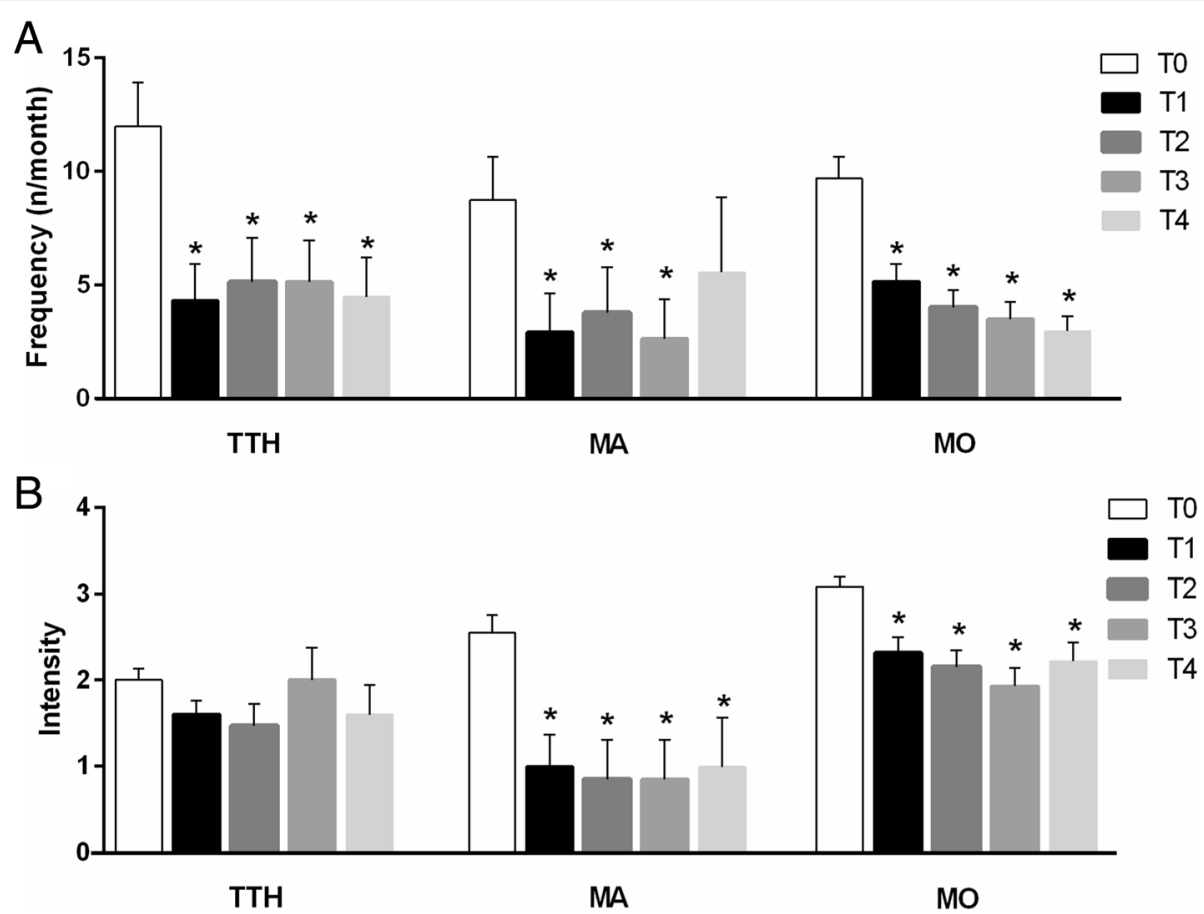

Fig. 1 Results for frequency of attacks (a) and Intensity of pain (b) at baseline and at the post-baseline time points by subgroup of diagnosis (values are means). Migraine with (MO) or without aura (MA), tension-type headache (TTH). Bars are standard deviations. ${ }^{*} p<0.05$

although not significantly, among MA respect to MO patients when evaluated during treatment administration (T2: $69 \%$ vs $29 \% ; p=0.12$ ) and at the end of the study (T4: $67 \%$ vs $23 \%$; $p=0.11$ ).

\section{Discussion}

Our study showed that administration of Partena ${ }^{\circ}$ for a period of 16 treatment weeks reduced headache frequency in a sample of children and adolescence affected by TTH and Migraine. Moreover, in our observational study, treatment with Partena was associated with lower intensity of pain for migraine episodes among pediatric patients suffering from MA and MO. We used a combination of magnesium, riboflavin, CQ10, Tanacetum parthenium, molecules all arising in the guidelines of the American Headache Society (AHS) and American Academy of Neurology (AAN) [14] in addition to the Andrographis paniculata (plant extract that has shown analgesic and anti-edema activity in preclinical studies along with anti-nociceptive effects in an animal model of sensory hypersensitivity associated with migraine) [15]. Guidelines of the Società Italiana Studio Cefalee (SISC) support the use of feverfew, $\mathrm{Mg}^{2+}$, riboflavin and CQ10 a Level III recommendation [16].

Tanacetum parthenium (feverfew) has been used for centuries to treat pain and headache [17]. Several preclinical studies have identified different targets for parthenolide like inhibition of platelet aggregation, inhibition of serotonin release from platelets and white blood cells, reduction of iNOS (inducible nitric oxide synthase) activation, inhibition of nuclear factor-kappaB (a transcriptional factor involved in the mediation of pain and inflammation) and binding to and inhibiting IKB kinase complex (IKK) $\beta$ that plays an important role in proinflammatory cytokine-mediated signaling $[18,19]$. More recent pharmacological data revealed that antimigraine effect of parthenolide may derives from its ability to target transient receptor potential ankyrin 1 (TRPA1), inducing nociceptor desensitization, ultimately resulting in inhibition of Calcitonin gene-related peptide (CGRP) release within the trigeminovascular system [20]. Clinical evidences about the treatment of migraine are still controversial although the contradictory results may be attributed to the variations in the concentration and to the differences in the stability of the parthenolides. The most recent randomized controlled trial, using a stable feverfew extract, added some positive evidences about the efficacy of parthenium in the prophylactic treatment of migraine [17, 21]. Studies on Feverfew efficacy in children and adolescents with migraine are lacking [11].

The other active principles of Partena ${ }^{\circ}$ currently available in Europe and USA as dietary supplements, have several epidemiologic, preclinical and clinical evidences supporting their usefulness in prophylactic treatment of migraine [21]. Several studies revealed decreased levels of the micronutrients riboflavin (Vit B2), magnesium (Mg) 
and CQ10 in plasma and brain of migraine patients [22]. On the other hand, numerous studies suggest a mitochondrial energy depletion in patients with migraine, and since all these molecules play an important role in the production of energy at mitochondrial level, multiple trials have assessed their efficacy in migraine prevention [23-25]. Riboflavin is a cofactor in oxidation-reduction reaction in the citric acid cycle and the electron transport chain, as such it plays a key role in the mitochondrial production of energy [21]. To date, evidence in headache treatment for pediatric age gave discordant results $[9,10,26]$. CQ10 is a vitamin-like compound needed for all cellular processes requiring energy. It is an electron-carrier, transferring electrons from complex I/complex II to cytochrome $\mathrm{C}$ in the mitochondrial electron transport chain [27]. Preliminary evidences appear to support efficacy for CQ10 in preventing migraines in children and adolescents, but remain insufficient to make a strong conclusion [26]. $\mathrm{Mg}^{2+}$ is necessary as a co-factor for proper functioning of the ATP-synthase. Furthermore, $\mathrm{Mg}^{2+}$ is the physiological antagonist at the NMDA-channel which is involved in the regulation of neuronal excitability and inhibition of cortical spreading depression (CSD) through several mechanisms involving serotonin receptors, NO synthesis/release as well as NMDA receptors [28]. The role of magnesium in the prophylactic treatment of pediatric migraine is still unclear and high-quality adequately powered trials are needed [26]. Andrographolide has a broad range of pharmacological properties, mainly an anti-inflammatory effect, by interfering with the production of inflammatory mediators, in particular cyclooxygenase (COX) enzymes and pro-inflammatory cytokines through the modulation of the NF-kB signaling network and the NO/iNOS pathway [29].

Nevertheless, it is conceivable to suppose that administration of key micronutrients and phytoextracts able to inhibit prostaglandin production and to interfere with the $\mathrm{NO} / \mathrm{iNOS}$ pathway as well as mitochondrial production of energy, might prevent and/or reduce the number and intensity of the attacks. This is more interesting when considering that treating headaches in pediatric population is a continuous challenge for clinicians. Although migraine in children is phenotypically similar to adult migraine, medications efficacious in adults did not show similar efficacy in pediatric controlled clinical trial and none are currently FDA-approved for this age group [30, 31]. Therefore, it may be necessary to use medications off label strictly weighing up the benefits and risks.

This study was performed on a small sample and the clinical effects of nutraceutical have not been compared with placebo. In fact, a limitation of our study is linked to the absence of a control group and on short time of observation.

\section{Conclusions}

To date there are scarce and contrasting evidences in favor of the most utilized drugs for prophylactic therapy in pediatric migraine and there are no significant differences between the high placebo response rate and the low drug response rate in pediatric age $[9,10]$. Thus, the adult model of headache treatment, in which, for example, amitriptyline and topiramate, have been effective, may not apply to pediatric patients. In addition, the reported serious adverse events in children, do not show a favorable risk-benefit profile for the use of these therapies in pediatric migraine prevention [9].

On the other hand, our result clearly indicates that Partena ${ }^{\circ}$ was able to reduce headaches frequency and pain intensity in children and adolescences suffering from migraine and TTH, therefore should be considered as a potential valuable prophylactic option for pediatric migraine. Although this study remains mainly observational, clear indications on the potential effectiveness, along with a favorable tolerability profile, of this combination on pediatric headache/migraine arose, and suggestion to perform further studies emerges to confirm the trend observed here. Randomized controlled trials are needed to further assess the effectiveness in nutraceuticals' use for migraine treatment in child and adolescent.

\section{Abbreviations \\ AAN: American Academy of Neurology; AHS: American Headache Society; Cl: Confidence Interval; ED: Emergency Department; FDA: food and drugs administration; IKK: inhibiting kinase complex; iNOS: inducible nitric oxide synthase; MA: Migraine Without Aura; MO: Migraine With Aura; NMDA: N- Methyl D-Aspartate; NO: nitric oxidase; OR: Odd Ratio; SD: Standard Deviation; SISC: Società Italiana per lo Studio delle Cefalee; TRPA1: transient receptor potential ankyrin 1; TTH: Tension-Type Headache}

\section{Acknowledgements}

We would like to thank sincerely all the Parents who gave the consent to enroll their Children in this observational study.

\section{Funding}

We used no funds for this study.

\section{Availability of data and materials}

The datasets used and/or analyzed during the current study are available from the corresponding author on reasonable request.

\section{Authors' contributions}

PP, MPV and FM conceptualized and designed the study, drafted the initial manuscript, interpreted data, reviewed and revised the manuscript and equally contributed to the work; MG, LM, MGL and FZ, participated in the design of the study, performed statistical analysis, collected and interpreted data. UR, PS and PP participated in the design of the study, interpreted data, reviewed and revised the manuscript, coordinated the study. All authors read and approved the final manuscript as submitted. Ethics approval and consent to participate The study was approved by the Ethics Committee of the Sant'Andrea Hospital according to the Declaration of Helsinki (as revised in Seoul, Korea, October 2008).

Ethics approval and consent to participate

Not applicable.

Consent for publication

All the Authors gave the consent for publication.

\section{Competing interests}

The authors declare that they have no competing interests. No financial or non-financial benefits have been received or will be received from any party related directly or indirectly to the subject of this article. No honorarium, grant, or other form of payment was given to anyone to produce the manuscript. 


\section{Publisher's Note}

Springer Nature remains neutral with regard to jurisdictional claims in published maps and institutional affiliations.

\section{Author details}

${ }^{1}$ Child \& Adolescent Neuropsychiatry, Hospital S. Orsola Malpighi, Bologna University, Bologna, Italy. ${ }^{2}$ Child Neurology, Pediatric Headache \& Sleep Disorders Centre, Chair of Pediatrics, NESMOS Department, Faculty of Medicine and Psychology, Sapienza University, Via Di Grottarossa, 1035-1039, 00189 Rome, Italy. ${ }^{3}$ Paediatric Neurology and Muscular Diseases Unit, Department of Neurosciences, Rehabilitation, Ophthalmology, Genetics, Maternal and Child Health, University of Genoa, 'G. Gaslini' Institute, Genoa, Italy. ${ }^{4}$ Child \& Adolescent Neuropsychiatry Unit, "Antonio Cao" Paediatric Hospital, "G.Brotzu" Hospital, Cagliari, Italy. ${ }^{5}$ Pediatric Headache Center, Neurology Unit Hospital, University of Sassari, Sassari, Italy. ${ }^{6}$ Pediatric Emergency Department, Bambino Gesù Children's Hospital, IRCCS, Rome, Italy.

Received: 8 January 2019 Accepted: 25 February 2019

Published online: 12 March 2019

\section{References}

1. Kacperski J, Bazarsky A. New developments in the prophylactic drug treatment of pediatric migraine: what is new in 2017 and where does it leave us? Curr Pain Headache Rep. 2017;21(8):38. https://doi.org/10.1007/ s11916-017-0638-4

2. Powers SW, Patton SR, Hommel KA, Hershey AD. Quality of life in paediatric migraine: Characterization of age-related effects using Peds QL 4.0. Cephalalgia. 2004:24:120-7.

3. Headache Classification Committee of the International Headache Society (IHS). The International Classification of Headache Disorders, 3rd edition. Cephalalgia. 2018 Jan;38(1):1-211.

4. Termine C, Ozge A, Antonacci F, Natriashvili S, Guidetti V, Wober-Bingol C. Overview of diagnosis and management of pediatric headache. Part II: therapeutic management. J Headache Pain. 2011;12:25-34.

5. Guidetti V, Galli F. Evolution of headache in childhood and adolescents: an 8-year follow up. Cephalalgia. 1998;18:449-54.

6. Orr SL, Kabbouche MA, O'Brien HL, et al. Paediatric migraine: evidence-based management and future directions. Nat Rev Neurol. 2018;14(9):515-27.

7. El-Chammas K, Keyes J, Thompson N, Vijayakumar J, Becher D, Jackson JL. A comparative effectiveness meta-analysis of drugs for the prophylaxis of pediatric migraine headache. JAMA Pediatr. 2013;167(3):250-8. https://doi. org/10.1001/jamapediatrics.2013.508.

8. Kacperski J, Kabbouche MA, O'Brien HL, Weberding JL. The optimal management of headaches in children and adolescents. Ther Adv Neurol Disord. 2016;9(1):53-68. https://doi.org/10.1177/1756285615616586.

9. Powers SW, Coffey CS, Chamberlin LA, Ecklund DJ, Klingner EA, Yankey JW et al. Trial of amitriptyline, topiramate, and place- Bo for pediatric migraine. N Engl J Med. 2017;376(2):115-24.

10. Evers S, Marziniak M, Frese A, Gralow I. Placebo efficacy in childhood and adolescence migraine: an analysis of double blind and placebo-controlled studies. Cephalalgia. 2009;29:436-44.

11. Sangermani R, Boncimino A. The use of nutraceutics in children's and adolescent's headache. Neurol Sci. 2017;38(Suppl 1):121-4.

12. Headache Classification Committee of the International Headache Society (IHS). The International Classification of Headache Disorders, 3rd edition (beta version). Cephalalgia. 2013;33:629-808.

13. Grinnon ST, Miller K, Marler JR, Lu Y, Stout A, Odenkirchen J, Kunitz S. National Institute of Neurological Disorders and Stroke Common Data Element Project approach and methods. Clin Trials. 2012;9(3):322-9 Epub 2012 Feb27.

14. Holland S, Silberstein SD, Freitag F, Dodick DW, Argoff C, Ashman E. Evidence-based guideline update: NSAIDs and other complementary treatments for episodic migraine prevention in adults: report of the quality standards Subcommittee of the American Academy of neurology and the American headache society. Neurology. 2012;78(17):1346-53.

15. Greco R, Siani F, Demartini C, et al. Andrographis paniculata shows antinociceptive effects in an animal model of sensory hypersensitivity associated with migraine. Functional Neurology. 2016;31(1):53-60. https://doi.org/10.11138/FNeur/2016.31.1.053.

16. Società Italiana per lo Studio delle Cefalee. Linee Guida per la diagnosi e la terapia delle cefalee primarie 2011.
17. Wider B, Pittler MH, Ernst E. Feverfew for preventing migraine. Cochrane Database Syst Rev. 2015, Issue 4. Art. No.: CD002286. DOl: https://doi.org/10, 1002/14651858.CD002286.pub3.

18. Kwok BH, Koh B, Ndubuisi Ml, Elofsson $\mathrm{M}$, Crews $\mathrm{CM}$. The anti-inflammatory natural product parthenolide from the medicinal herb feverfew directly binds to and inhibits lkappaB kinase. Chem Biol 2001;8(8):759-766. PubMed PMID: 11514225

19. Tassorelli C, Greco R, Morazzoni P, Riva A, Sandrini G, Nappi G. Parthenolide is the component of Tanacetum parthenium that inhibits nitroglycerininduced Fos activation: studies in an animal model of migraine. Cephalalgia: An International Journal of Headache. 2005;25:612-21.

20. Materazzi S, Benemei S, Fusi C, et al. Parthenolide inhibits nociception and neurogenic vasodilatation in the trigeminovascular system by targeting TRPA1 channel. Pain. 2013;154(12):10.

21. Daniel O, Mauskop A. Nutraceuticals in acute and prophylactic treatment of migraine. Curr Treat Options Neurol. 2016:18(4):14.

22. Taylor FR. Nutraceuticals and headache: the biological basis. Headache. 2011:51(3):484-501.

23. Cevoli S, Favoni V, Cortelli P. Energy metabolism impairment in migraine. Curr Med Chem. 2018. https://doi.org/10.2174/0929867325666180622154411 [Epub ahead of print].

24. Markley HG. CoEnzyme Q10 and riboflavin: the mitochondrial connection. Headache. 2012;52(Suppl 2):81-7.

25. Sándor PS, Di Clemente L, Coppola G, Saenger U, Fumal A, Magis D, Seidel L, Agosti RM, Schoenen J. Efficacy of coenzyme Q10 in migraine prophylaxis: a randomized controlled trial. Neurology. 2005;64(4):713-5.

26. Orr SL. The evidence for the role of nutraceuticals in the Management of Pediatric Migraine: a review. Curr Pain Headache Rep. 2018;22(5):37.

27. Miles MV, Morrison JA, Horn PS, Tang PH, Pesce AJ. Coenzyme Q10 changes are associated with metabolic syndrome. Clin Chim Acta. 2004;344(1-2):173-9.

28. Sun-Edelstein C, Mauskop A. Role of magnesium in the pathogenesis and treatment of migraine. Expert Rev Neurother. 2009;9(3):369-79.

29. Akbar S. Andrographis paniculata: a review of pharmacological activities and clinical effects. Altern Med Rev. 2011;16(1):66-77.

30. Blume HK. Childhood headache: a brief review. Pediatr Ann. 2017;46(4):e155-65.

31. Hershey AD, Powers SW, Coffey CS, et al. Childhood and adolescent migraine prevention (CHAMP) study: a double-blinded, placebo-controlled, comparative effectiveness study of amitriptyline, Topiramate and placebo in the prevention of childhood and adolescent migraine. Headache. 2013; 53(5):799-816. https://doi.org/10.1111/head.12105.

Ready to submit your research? Choose BMC and benefit from:

- fast, convenient online submission

- thorough peer review by experienced researchers in your field

- rapid publication on acceptance

- support for research data, including large and complex data types

- gold Open Access which fosters wider collaboration and increased citations

- maximum visibility for your research: over $100 \mathrm{M}$ website views per year

At BMC, research is always in progress.

Learn more biomedcentral.com/submissions 